\section{Tamizaje del cáncer colorrectal ${ }^{1}$}

Palabras clave: cáncer colorrectal, colonoscopia, enema de bario, sangre oculta en heces, sigmoidoscopia.

\footnotetext{
Basado en el documento "Screening for Colorrectal Cancer. Recommendations and rationale", elaborado por el Grupo de Trabajo Especial sobre Servicios Preventivos de Estados Unidos (U.S. Preventive Services Task Force), disponible a texto completo, en inglés, en: http:// www.ahcpr.gov/clinic/3rduspstf/colorectal/colorr.htm
}

\section{EPIDEMIOLOGÍA DEL CÁNCER COLORRECTAL}

El cáncer colorrectal (CCR) es el cuarto cáncer más frecuente y la segunda causa de muerte por cáncer en Estados Unidos (EE. UU.).

Más del $80 \%$ de los CCR aparecen en pólipos adenomatosos, cuya prevalencia aumenta del 20 a $25 \%$ a los 50 años a $50 \%$ a los 75 a 80 años.

Los pólipos adenomatosos de menos de $1 \mathrm{~cm}$ que acaban sufriendo transformación maligna son menos del $1 \%$, pero el $10 \%$ de los mayores de $1 \mathrm{~cm}$ acaba malignizándose a los 10 años, y el 25\% a los 20 años.

La mayoría de los CCR ocurren en personas con riesgo medio, pero $20 \%$ ocurren en pacientes con factores de riesgo específicos, como los antecedentes de CCR en familiares en primer grado.

Cerca del 6\% de los casos se asocian a síndromes genéticos poco frecuentes, como la poliposis adenomatosa familiar o el CCR no polipósico hereditario.

El riesgo también está aumentado en pacientes con colitis ulcerosa de larga duración, en los ya diagnosticados de grandes pólipos adenomatosos o CCR y en aquellos con antecedentes familiares de pólipos adenomatosos diagnosticados antes de los 60 años.

\section{PRUEBAS DISPONIBLES}

Todas las pruebas disponibles para el tamizaje del CCR (cuadro 1) tienen ventajas e inconvenientes que varían según el paciente y el medio.

La elección debe basarse en las preferencias del paciente y en su adherencia a las recomendaciones del médico, en las contraindicaciones médicas y en los recursos disponibles tanto para la realización de las pruebas como para el seguimiento de los pacientes.

El paciente debe ser informado de los beneficios y riesgos de cada prueba.

\section{Intervalo entre pruebas}

El intervalo óptimo entre pruebas depende de cuáles se utilicen. 
CUADRO 1. Pruebas para el tamizaje del cáncer colorrectal

Detección de sangre oculta en las heces (DSOH)a

Sigmoidoscopia con endoscopio flexible

Combinación de DSHO y sigmoidoscopia

Colonoscopia

Enema de bario con doble contraste

a Realizada en el domicilio del paciente, en múltiples muestras consecutivas.

La detección de sangre oculta en las heces (DSOH) una vez al año proporciona una mayor reducción de las tasas de mortalidad que la $\mathrm{DSOH}$ bienal, pero el número de resultados falsamente positivos también es mayor.

No hay datos que permitan determinar el intervalo óptimo entre otras pruebas.

\section{Edad de inicio e interrupción de las pruebas}

Hay pocos datos para determinar la edad óptima de inicio y de interrupción del tamizaje del CCR.

La DSOH ha demostrado ser eficaz en personas de 50 a 80 años, y la sigmoidoscopia se ha asociado a una disminución de la mortalidad en mayores de 45 años.

En pacientes con un riesgo medio de CCR se recomienda iniciar el tamizaje a los 50 años.

Es razonable iniciarlo antes en individuos con mayor riesgo, como aquellos con familiares en primer grado con CCR antes de los 60 años.

Existen directrices clínicas para la realización de estas pruebas en pacientes de muy alto riesgo, como los que tienen antecedentes de poliposis familiar, CCR no polipósico hereditario o colitis ulcerosa de larga duración.

En pacientes con síndromes genéticos puede estar indicada la realización precoz de colonoscopias y pruebas genéticas.

No se sabe a qué edad se deben interrumpir las pruebas de tamizaje, pero los estudios realizados se han limitado generalmente a los menores de 80 años.

\section{Detección de sangre oculta en las heces (DSOH)}

Para el tamizaje mediante DSOH se recomienda la obtención de tres muestras de heces consecutivas. La rehidratación de las muestras antes del análisis aumenta la sensibilidad, pero también aumenta considerablemente el número de resultados falsamente positivos. No se recomienda el tacto rectal ni el uso de una sola muestra obtenida durante el tacto rectal.

\section{DSOH y sigmoidoscopia}

La combinación de ambas puede detectar más cánceres y más pólipos grandes $(>1 \mathrm{~cm})$ que cualquiera de las dos pruebas por separado, pero no se conocen bien los beneficios adicionales y los posibles riesgos de su uso combinado.

En general, la DSOH debe preceder a la sigmoidoscopia, puesto que la obtención de un resultado positivo indica la realización de una colonoscopia, evitándose así la sigmoidoscopia.

\section{Colonoscopia}

La colonoscopia es la prueba más sensible y específica para detectar cánceres y pólipos grandes, pero también conlleva mayores riesgos que las demás pruebas.

No es seguro que los potenciales beneficios adicionales de la colonoscopia en comparación con las otras pruebas justifiquen los riesgos adicionales y las molestias para el paciente.

Los costos iniciales de la colonoscopia también son mayores que los de las demás pruebas.

\section{EXACTITUD Y FIABILIDAD DE LAS PRUEBAS}

\section{Tacto rectal y DSOH en la consulta}

Hay pocos datos para determinar la eficacia del tacto rectal o de la DSOH realizada en la consulta con una muestra aislada de heces obtenida mediante tacto rectal.

De cualquier modo, se sabe que los CCR al alcance del dedo explorador son menos de $10 \%$.

La sensibilidad de una única DSOH realizada en la consulta es probablemente bastante inferior a la conseguida con múltiples DSOH realizadas en el domicilio del paciente.

\section{DSOH}

La sensibilidad de la DSOH depende del protocolo.

La sensibilidad y especificidad estimadas de una sola prueba son de $40 \%$ y 96 a $98 \%$, respectivamente.

La rehidratación de las muestras aumenta la sensibilidad $(60 \%)$, pero reduce la especificidad (90\%).

En un estudio, el tamizaje anual con rehidratación de las muestras detectó $49 \%$ de los nuevos cánceres. Las muestras no rehidratadas y las prue- 
bas bienales detectan una menor proporción de cánceres (27 a 39\%), pero requieren menos colonoscopias (5 a $28 \%$ ).

\section{Sigmoidoscopia}

La primera sigmoidoscopia detecta unos 7 cánceres y unos 60 pólipos grandes o de alto riesgo por cada 1000 exámenes.

Aunque es difícil cuantificar su tasa de falsos positivos, el tamizaje endoscópico puede llevar a la extirpación de muchos pólipos con bajo potencial de malignidad o que no se habrían manifestado clínicamente.

\section{DSOH y sigmoidoscopia}

Se ha propuesto la combinación de la DSOH con sigmoidoscopias periódicas como forma de aumentar la sensibilidad.

En tres ensayos clínicos aleatorizados recientes, la sigmoidoscopia tras la DSOH permitió diagnosticar unos 7 cánceres o pólipos grandes adicionales por cada 1000 exámenes, en comparación con la DSOH aislada. En cambio, la DSOH tras la sigmoidoscopia no aumentó el número de casos diagnosticados por esta.

\section{Enema de bario con doble contraste (EBDC)}

La mayoría de los estudios sobre esta prueba tienen importantes limitaciones que no permiten determinar su exactitud en poblaciones asintomáticas.

En estudios anteriores se registró una alta sensibilidad (86 a $90 \%$ ) y especificidad (95\%), pero en el National Polyp Study el EBDC solo detectó 48\% de los pólipos de más de $1 \mathrm{~cm}$.

\section{Colonoscopia}

Recientemente se ha defendido el uso de la colonoscopia como prueba de tamizaje, bien a intervalos de 10 años, o bien una sola vez en la vida, a $\operatorname{los} 55$ a 65 años.

Resulta difícil determinar la exactitud de la colonoscopia, puesto que se suele utilizar como prueba de referencia.

La sensibilidad estimada de una colonoscopia aislada es de $90 \%$ para los pólipos grandes y de $75 \%$ para los pequeños.

Muchos pacientes son sometidos a la extirpación de pólipos durante la colonoscopia, aunque solo una minoría de ellos se habrían malignizado.

\section{Colografía por tomografía computadorizada (CTC)}

Esta prueba puede ser relativamente sensible y específica ( 85 a $90 \%$ ) cuando se practica en el contexto de investigaciones, pero informes recientes indican que es menos exacta cuando la practican examinadores con menos experiencia.

Los pólipos pequeños y los pólipos planos se visualizan peor que los cánceres y los pólipos grandes.

\section{EFICACIA DE LA DETECCIÓN PRECOZ}

\section{DSOH}

Tres estudios aleatorizados controlados han mostrado que la $\mathrm{DSOH}$ periódica reduce el riesgo de muerte por CCR en un 15 a $33 \%$.

\section{Sigmoidoscopia}

Los datos existentes proceden únicamente de estudios de casos y controles, pero están en marcha dos estudios aleatorizados controlados que proporcionarán resultados dentro de unos 5 años.

Un estudio de casos y controles con un gran número de participantes indicó que la sigmoidoscopia reduce en $59 \%$ el riesgo de muertes por CCR localizados al alcance del sigmoidoscopio rígido.

\section{DSOH y sigmoidoscopia}

No se han realizado estudios aleatorizados controlados para determinar si esta combinación reduce la mortalidad o la morbilidad más que cualquiera de las dos pruebas por separado.

En un estudio controlado no aleatorizado, al añadir la DSOH se detectaron más cánceres en el tamizaje inicial que al usar solo la sigmoidoscopia rígida, pero la mortalidad a los 9 años no fue significativamente menor. No se sabe si estos resultados son generalizables a la sigmoidoscopia flexible.

\section{Colonoscopia}

No se han realizado estudios aleatorizados controlados sobre la eficacia de la colonoscopia para reducir la morbilidad o la mortalidad del CCR.

En el National Polyp Study se estimó que la colonoscopia periódica podría prevenir 76 a 90\% de los cánceres. 


\section{EBDC y CTC}

Todavía no se han realizado estudios sobre la eficacia de estas pruebas para reducir la mortalidad o la morbilidad del CCR.

\section{RIESGOS DEL TAMIZAJE}

La DSOH tiene escasos riesgos, pero los falsos positivos pueden llevar a la realización de procedimientos innecesarios, como la colonoscopia.

La sigmoidoscopia puede producir perforación intestinal, aunque se trata de una complicación rara ( 1 a 2 casos por 10000 exámenes).

El EBDC también tiene pocas complicaciones importantes (1 por 10000 exámenes), entre ellas la perforación intestinal (1 por 25000$)$ y la muerte (1 por 55000 ).

La colonoscopia de tamizaje tiene más riesgos que la DSOH o la sigmoidoscopia, riesgos que son todavía mayores cuando también se usa para procedimientos terapéuticos, como la polipectomía. En dos estudios de colonoscopias de tamizaje en más de 5000 pacientes, 0,2 a $0,3 \%$ de ellos sufrieron complicaciones durante la prueba o inmediatamente después, siendo la más frecuente la hemorragia grave, con necesidad de hospitalización o tratamiento de urgencia. CTC.

No hay datos sobre las complicaciones de la

\section{PREFERENCIAS Y ADHERENCIA DE LOS PACIENTES}

De 50 a $70 \%$ de los pacientes completan la DSOH cuando así se lo aconseja el médico.

La adherencia a la sigmoidoscopia inicial es del 25 a $50 \%$, pero no hay datos con respecto a su repetición.

En un estudio, después de informarlos sobre las pruebas y darles a elegir entre la DSOH aislada, la sigmoidoscopia y la combinación de ambas pruebas, la mayoría de los pacientes optaron por la DSOH aislada o ambas pruebas, y solo el 8 a 13\% prefirió la sigmoidoscopia aislada.

Sin embargo, la adherencia a la combinación de ambas pruebas es menor que la adherencia a cualquiera de ellas por separado.

No se ha investigado la aceptación del EBDC por parte de los pacientes.

\section{COSTO-EFECTIVIDAD}

Las estimaciones realizadas indican que, desde el punto de vista social y en comparación con la ausencia de tamizaje, todos los métodos de tamizaje del CCR presentan una relación costo-efectividad tan buena como muchos otros servicios clínicos preventivos: menos de 30000 dólares estadounidenses por año de vida ganado.

Hay divergencias con respecto a la estrategia con mejor relación costo-efectividad.

\section{RECOMENDACIONES DE OTRAS ORGANIZACIONES}

Las recomendaciones de la American Cancer Society se resumen en el cuadro 2. El American College of Surgeons, el American College of Obstetricians and Gynecologists, la American Academy of Family Physician, y la American Gastroenterological Association han hecho recomendaciones similares.

CUADRO 2. Recomendaciones de la American Cancer Society para el tamizaje del cáncer colorrectal en mayores de 50 años con riesgo normal

Detección de sangre oculta en las heces (DSOH) anual Sigmoidoscopia flexible cada 5 años

DSOH anual y sigmoidoscopia flexible cada 5 años

Enema de bario con doble contraste cada 5 años

Colonoscopia cada 10 años

\section{CONCLUSIONES}

- Recomendar firmemente a los médicos que les realicen pruebas de tamizaje del CCR a sus pacientes de ambos sexos con 50 años o más.

- Hay buenas pruebas de que varios métodos de tamizaje son eficaces para reducir la mortalidad por CCR.

- Los beneficios del tamizaje superan sus riesgos, aunque la calidad de las pruebas, la magnitud del beneficio y los riesgos varían según el método.

La Canadian Task Force on Preventive Health Care ha concluido que hay buenos datos para recopara recomendar la sigmoidoscopia como parte de los exámenes periódicos de adultos de más de 50 la colonoscopia o la combinación de la DSOH con la sigmoidoscopia. 
- Hay buenas pruebas de que la DSOH periódica reduce la mortalidad por CCR y pruebas aceptables de que la sigmoidoscopia, aislada o combinada con la DSOH, tiene el mismo efecto.

- No hay pruebas directas de que la colonoscopia de tamizaje sea eficaz para reducir la mortalidad por CCR, aunque su eficacia es apoyada por otros tipos de datos.

- No está claro que la mayor exactitud de la colonoscopia en comparación con las demás pruebas compense sus complicaciones, molestias y costos adicionales.

- El EBDC es menos sensible que la colonoscopia y no hay pruebas de que sea eficaz para reducir la mortalidad.

- No hay pruebas suficientes de que técnicas más recientes, como la CTC, consigan mejorar los desenlaces sanitarios.

- No hay datos suficientes para determinar cuál es el mejor método de tamizaje, tanto desde el punto de vista de los beneficios y riesgos como de la relación costo-eficacia.

- Es probable que el tamizaje del CCR tenga buena relación costo-efectividad (menos de 30000 dólares estadounidenses por año de vida ganado), independientemente del método elegido.

\section{SYNOPSIS}

\section{Screening for colorectal cancer}

This piece summarizes new recommendations from the Preventive Services Task Force of the United States of America concerning screening for colorectal cancer (CRC). These recommendations update and replace ones that were issued in 1996. The Task Force strongly recommends that physicians carry out CRC screening tests for both men and women who are 50 years of age or older. The Task Force found fair or good evidence that: 1) several screening methods are effective in reducing mortality from $C R C, 2)$ the benefits of screening outweigh its risks, although the quality of the tests, the magnitude of the benefits, and the potential harms vary according to the method, and 3) periodic fecal occult blood testing (FOBT) reduces mortality from CRC. In addition, there is fair evidence that sigmoidoscopy, either alone or in combination with FOBT, reduces CRC mortality. There is no direct evidence that screening colonoscopy is effective in reducing CRC mortality, nor is it clear if the greater accuracy of colonoscopy in comparison to other tests compensates for its additional complications, inconvenience, and costs. Doublecontrast barium enema is less sensitive than colonoscopy, and there is no direct evidence that it is effective in lowering mortality rates. There are insufficient data to determine which screening strategy is best in terms of the balance of benefits, potential harms, and cost-effectiveness. Regardless of the strategy chosen, CRC screening is likely to be costeffective (less than US\$30 000 per year of life gained).

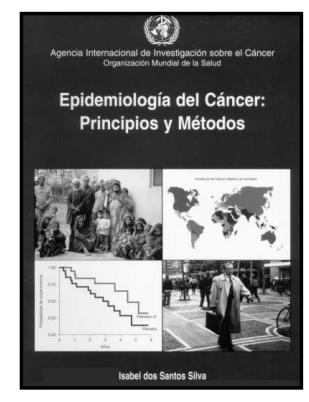

\section{Epidemiología del cáncer: principios y métodos}

Esta obra se dirige principalmente a estudiantes de medicina y salud pública, médicos, profesionales de la salud y cualquiera interesado enn entender los principios y métodos aplicados en la epidemiología del cáncer. Su objetivo no es formar expertos, sino instruir a los lectores en el empleo de herramientas epidemiológicas básicas y hacerlos capaces de ejercer un juicio crítico cuando evalúan los resultados publicados por otros.

La obra está diseñada para proporcionar - mediante ejemplos ilustrativos y, con frecuencia, datos obtenidos mediante investigaciones - información fácilmente comprensible sobre los conceptos básicos y métodos de investigación en el campo de la epidemiológica.

1999• 471 pp. ISBN 9283204077 • Código: OT 113• Precio: \$US 40.00/ 32.00 en países de América Latina y el Caribe

Para adquirir esta publicación: http://publications.paho.org; E-mail: paho@pmds.com; Fax: (301) 209-9789; Tel: (301) 617-7806; (1-800) 472-3046 